\title{
Getting from Situations to Emotions: Appraisal and Other Routes
}

\author{
Brian Parkinson \\ Oxford University, UK
}

\begin{abstract}
When processing situational information, people can reach emotional conclusions without explicitly registering corresponding appraisals. Does this mean that appraisal cues must be guiding inference in less obvious ways? Assuming that the emotional meaning of any situation depends on the protagonist's relation to what is happening, then emotion inference can never entirely bypass relational information. However, not all relational information is specifically appraisal-based. Further, actual emotion causation, like emotion inference, can involve explicit or implicit appraisals, or even no appraisals at all. Indeed, humans do not first learn to associate emotions with situations by extracting appraisal information.
\end{abstract}




\section{Getting from Situations to Emotions: Appraisal and Other Routes}

You are stepping onto the bus when your carrier bag splits apart, scattering groceries across the pavement. What emotion do you feel? Is this event desirable? Who or what is to blame? Knowing the answers to any of these questions seems to help with some of the others, so there must be connections between them. But what kind of connections? Which questions, those relating to appraisals or those relating to emotions, are more fundamental in meaning apprehension? Siemer and Reisenzein make innovative use of reaction-time methodology to address these issues, then proceed to apply their conclusions to the broader question of how actual rather than described situations produce occurrent rather than inferred emotions. For them, there are connections here too, because emotions are based on proposition-like appraisals that are implicated at some level regardless of whether the information they encapsulate is presented as real-time, responsive, multimodal stimulation or as words on a computer screen. In both cases, they believe that it is information that counts in emotion differentiation rather than the pushes and pulls of unfolding transactions.

This commentary evaluates Siemer and Reisenzein's conclusion that emotion inference from situation descriptions depends on processing of appraisal information. Although many of their findings may be open to alternative explanations, it seems noncontroversial that some descriptions of emotional events (just like some actual emotional events) only lead to emotional implications once appraisal-relevant relational features are registered. More precisely, we sometimes need clarification of the protagonist's orientation towards an event before we can work out what emotion is appropriate. Nevertheless, none of this implies that appraisal mediation is the original, most basic, or most familiar path from a situation (or situation description) to an emotion (or emotion inference). 
Emotion Inference Without Appraisal Inference: Study 1

In study 1 , Siemer and Reisenzein provide further support for a conclusion that at first seems a direct refutation of some extensions of appraisal theory. Even when only situational information is presented, participants arrive at emotion judgements more quickly than appraisal judgements (see also Schuster, Rudolph, \& Försterling, 1998). At minimum, this shows that participants cannot be making explicit inferences in the form of the presented appraisal items en route to their emotional inferences. For instance, participants don't need to have said to themselves "I experience this as undesirable," "this is unfair," and "other people/circumstances are responsible" before being able to affirm the truth of "I experience the following emotion: Anger." Otherwise they would be able to respond to each of the former items at least as quickly as to the last one.

But the fact that participants do not formulate appraisal inferences in these specific terms does not necessarily mean that their emotion conclusions are independent of any kind of appraisals. One possibility is that both emotions and appraisals are inferred at an implicit level but the representations of emotion are more easily translated into the verbal descriptions provided. Deciding whether one's disliking of events matches the undesirability item may take longer than deciding whether one's sense of anger matches the corresponding emotion item. ${ }^{1}$ More generally, it may be that emotion concepts are closer to spontaneous representations of situations (more "experience-near") than technical appraisal concepts.

Siemer and Reisenzein take a different tack. For them, the propositional appraisals specified in the questions do match the original form and content of participants' underlying appraisal representations. However, these representations have been used so often to get to a corresponding emotion that they have become automatic and can no longer be accessed directly (indeed, they may even be bypassed completely due to knowledge compilation, e.g., Anderson, 1987). An analogy might be that thinking about the specific movements involved 
in changing gear while driving often interferes with the performance of this skilled action (cf. Vallacher, Wegner, \& Somoza, 1989). Nevertheless, habitual gear-changing still depends on implicitly registering the same cues that were originally used when first learning to drive.

Although I agree that emotion inference from textual description is often a skilled and partly automatic process for most adults, I see no evidence that this process was originally acquired by repeatedly extracting explicit appraisals. Instead, appraisal representations are themselves abstractions and articulations of more fundamental relational dynamics (Barrett, 1995). A young infant cries while struggling against the physical resistance of an arm restraining her movements (e.g., Watson, 1929). Only later does she begin to direct an accusatory gaze to the face of whoever is restraining her. Later still, this implicit other-blame appraisal (Lazarus, 1991) is explicitly linked to notions of accountability (see Parkinson, Fischer, \& Manstead, 2005). In short, emotions first emerge as direct adjustments to relational dynamics, not articulated responses to propositional representations of appraisal information.

From this angle, the most obvious explanation of study 1's findings is that participants read off emotions from the image they construct of the described event more directly than they read off appraisals. When thinking about how I might feel when my bag splits, for example, I picture myself in the scene and the frustrating character of what happens becomes immediately apparent. Only then might I conclude that this event is undesirable and seems unfair from that imagined perspective.

What Information is Accessed During Emotion Inference? Studies 2 and 3

Studies 2 and 3 attempt to demonstrate that appraisal information is implicated in emotion inference even if it is not encoded explicitly. Both experiments use a judgementfacilitation paradigm (e.g., Smith \& Miller, 1983) and work from the plausible assumption that responses are faster if relevant information has already been accessed to answer previous 
questions. Thus, if participants' appraisal judgements are faster after making emotion judgements, one explanation is that accessing emotion-relevant features of narratives also involves accessing appraisal-relevant features. For example, having worked out that a sentence implies anger, participants find it easier to conclude that it also implies external responsibility because they already made use of responsibility-related information to infer anger.

Apart from some interesting anomalies discussed later, Siemer and Reisenzein's results generally confirm that emotion judgements facilitate subsequent appraisal judgements, and appraisal judgements facilitate subsequent emotion judgements (possibly to a lesser extent than vice versa ${ }^{2}$ ) even after controlling for question sequence and emotion concept priming. This pattern is broadly consistent with Siemer and Reisenzein's hypotheses, but is their explanation the best one?

\section{Question clarification}

As outlined above, study 1's findings may be explained by the relative ease of processing emotion items in comparison with appraisal items. Although comparisons between latencies of first-position appraisal and emotion judgements in studies 2 and 3 are susceptible to similar explanations (see footnote 1), their facilitation effects are clearly not because the same items elicit quicker responses when presented in one sequence than when presented in another. Does this mean that question interpretation played no role in judgement facilitation?

One unconsidered possibility is that prior emotion questions clarify subsequent appraisal questions' relevance to the situation under consideration. For example, if asked whether someone else is responsible when "One of your dinner guests comments negatively on your cooking abilities" you may be unsure whether the question relates to what your guest says or your own cooking. However, if you have previously been asked whether you feel 
angry, the focus of the subsequent appraisal item becomes more obvious. Similarly, having reached the conclusion that you feel guilty when: "You tell people your friend orchestrated a prank: He is punished for it," makes it easier to see that appraisal questions apply to your friend getting punished rather than your original decision to expose your friend. ${ }^{3}$

Siemer and Reisenzein's results include indirect evidence for this last effect. As they state: "Guilt scenarios were more often evaluated as negative when the evaluation followed a guilt judgment than when it was made before this judgment." In other words, making the guilt judgement clarifies the situation's undesirability, maybe by focusing attention on the wrong done rather than the doing of this wrong. Perhaps this increased clarity made responses faster as well as more consistent.

A similar principle may also explain facilitation of emotion judgements by prior appraisal judgements. For instance, questions about emotions experienced when "you ridicule a friend in his presence ..." may refer to feelings that prompted ridicule or feelings about ridicule's effects on your friend. However, a prior question about personal responsibility makes it easier to recognise that you are being asked about responses to your action's consequences rather than the emotional impulse that motivated your action in the first place.

\section{Processing of situational information}

Are all of the reported response-facilitation effects amenable to interpretation in terms of question clarification? Probably not. Answering emotion questions probably also drew participants' attention to features of some of the described situations that were also relevant to answering subsequent appraisal questions (and vice versa), just as Siemer and Reisenzein claim. However, two important interpretational issues remain. Does appraisal-relevant information mediate all cases of emotion inference from situational information or only 
some? And does this information influence emotion inference specifically by virtue of its implications for appraisal?

Representativeness of vignettes. Is it possible to convey emotional meaning using situation descriptions that don't imply appraisal at some level? The answer partly depends on what counts as a situation description (and partly on what counts as appraisal). Obviously descriptions of facial expressions, bodily symptoms, and action tendencies are disallowed because they permit emotion inferences based on reactions to events rather than events themselves. Correspondingly, some explicit descriptions of appraisals (e.g., "she felt he was to blame for letting her down") might lead readers to emotional conclusions because the selective interpretation is seen as content or consequence rather than cause of emotion.

We might also want to rule out sentences such as "someone is being annoying" or "something frightening happens" because the words used to describe the external object as emotionally provocative already specify the protagonist's likely reaction. But couldn't we also register emotion-inducing properties from other less direct descriptions of the emotional affordances of an event such as "someone is pointing a gun at you" or "a wild animal is loose in the room"? Are appraisal cues also implicated here?

In these examples, emotion inference involves classifying objects in directly emotional terms rather than unpacking individual appraisals. However, perhaps relatively few emotions can be implied simply using simple object descriptions of this kind. Unless we know something about the protagonist's orientation to the object (relational information), an object's emotional effects often remain uncertain. Clearly one way of specifying this orientation is by presenting appraisal information (e.g., compare "she thought it unfair that he earned more than she did" to "she was pleased that he earned more than she did"), but is it the only way? 
Consider the following description of an emotion-inducing procedure used in an experiment by Stemmler (1989, p. 622), which has been edited to exclude information specifying the participant's reaction to (and interpretation of) what is happening:

"Anagrams were displayed for $5 \mathrm{~s}$, and the first 15 items were solvable. Then ..., the task was interrupted [by the experimenter], 'Listen, we can hardly understand you, although the amplifier's volume is already turned up to maximum. It would be best if you could speak louder!' The following anagram was solvable. Then ..., a second interruption, 'Louder, please!' The next anagram was unsolvable. After the subject's answer, ... [the experimenter says] 'Can't you speak up?",

Although information relating to external responsibility, unfairness, and undesirability is available in this narrative, is it attention to these aspects that underlies the inference of anger? Or is it instead that the interruptions and difficult anagrams seem directly frustrating when perceived from the described perspective?

Active ingredients of vignettes. Deriving emotional meanings from situation descriptions typically requires the use of information concerning the actor's relation to what is happening rather than just information about external events themselves. Does this already mean that appraisal information is accessed at some level? For example, does registering the "scariness" of a gunman already count as picking up appraisal cues? If so, the central question addressed by the present research lacks empirical content. If every kind of emotionrelevant situational information necessarily counts as appraisal information, then Siemer and Reisenzein need not have conducted such complicated studies to demonstrate that appraisal information mediates emotion inference.

For me, appraisal information constitutes only a subcategory of relational information, and relational information of other kinds can also mediate emotion inference from situation descriptions. This claim rests on a subtle but important distinction. When interpreting vignettes, participants might attend to protagonists' representations of their orientation to the situation (whether they perceive it as desirable/undesirable, whether they believe that external factors are responsible, whether they think it is unfair and so on) or they might use this 
imagined orientation to infer emotion more directly. Having put oneself in the position of someone getting on a bus (on the way somewhere) a carrier bag splitting open is directly perceived as frustrating (i.e., anger-provoking), just as a gunman is directly perceived as scary.

These two interpretations are easily confused because in both cases participants are working with representations of events rather than events themselves, and are making judgements about a projected self in second-person narratives rather than about someone whose mental processes are more obviously inaccessible. However, there is still a difference between the claim that a represented escalating obstacle to a current line of action leads to an anger inference, and the claim that a representation of a projected self's apprehension of an escalating obstacle leads to an anger inference.

But Siemer and Reisenzein do not claim that any actual appraisal inference mediates emotion inference, only that appraisal-relevant features must be accessed. So the only remaining difference between the two accounts concerns the basis for the relevance of relational information. Either participants used represented relational cues to infer emotion directly or they used these same cues because they were once associated with a now-absent appraisal inference that in turn implied an emotion inference. To me, the former model seems a simpler and more direct explanation for the relative slowness of appraisal judgements compared with emotion judgements across all three of the studies reported here. In either case, all that studies 2 and 3 really tell us is that emotion inferences and appraisal inferences rely on overlapping relational features in the presented stories, not how or why these relational features implied emotional conclusions.

Judgement Facilitation as a Method for Clarifying Emotion Concepts Regardless of the specific interpretation of their findings, Siemer and Reisenzein's procedure provides a potentially useful technique for determining which characteristics of 
represented transactions are associated with differential emotion concepts. The results certainly suggest that people attend to certain kinds of relational information when inferring particular emotions from these particular situation descriptions. Unfortunately, the presented sentences do not provide a representative selection of emotional situations. In order to demonstrate that these and only these relational features are relevant, it is necessary to sample more widely from possible occasions for each emotion, and to compare their proposed appraisal questions with alternatives.

Despite the restricted range of situational information provided in these studies, the predicted emotion was still not always inferred from the relevant situation description, did not always prime, and was not always primed by, the relevant appraisal judgement. In other words, participants sometimes drew emotional conclusions without using information directly relevant to the predicted appraisals.

For example, anger was inferred from situation descriptions intended to imply guilt in more than $50 \%$ of cases in study 1 . On what basis did participants arrive at this particular emotion inference? Not by extracting information relevant to external responsibility since no such information was specified. Correspondingly, only about half of responses to situations actually intended to imply anger endorsed external responsibility (see Siemer and Reisenzein's Table 1). So how did participants arrive at their anger inferences in these cases?

Even when information relating to the external responsibility item was explicitly accessed in study 2 , there was no subsequent facilitation of anger judgements. Finally, prior anger questions made no further difference to latency of responsibility judgements after the relevant emotion concept had been primed in Study 3, suggesting that any facilitation effect depended on implicit associations between anger and other-responsibility regardless of story processing. My conclusion from this coherent pattern of findings is that anger inference does not depend on extraction of information relating to external responsibility even when 
such information is specified in stimulus materials. This particular appraisal does not seem to be a necessary condition for attributed anger (see also Kuppens et al., 2003; Parkinson, 1999).

Getting from Real Situations to Real Emotions

I have argued that inferring emotion from situation descriptions often depends on extracting relational information that is also extracted when making appraisal judgements, and that identifying the specific kinds of information that are accessed helps to clarify emotion concepts and their application to situation descriptions. But what do Siemer and Reisenzein's results tell us about actual emotional reactions to events?

Sometimes our awareness of emotional situations is provided by someone else's description rather than direct personal experience. On some of these occasions the information is imparted in written text (e.g., an email or letter). In order to appreciate the emotional implications of the described event in these cases, we certainly need to extract information that specifies its relational meaning at some level. But what about situations that we confront more directly, when what is happening unfolds in relation to our active engagement with it?

Siemer and Reisenzein argue that "even when information about emotion-eliciting events is obtained through personal observation, this information may ultimately come to be represented in the same format (e.g., a propositional situation model) as the information conveyed by direct verbal communications." If this is correct, the findings from their studies have direct bearing on emotion causation itself, because both emotion inference and emotional experience depend on converting events (or their descriptions) into similar internal representations.

But is the only way of getting from situations to emotions via proposition-like appraisals of this kind? Is real life always directly analogous to a story that needs to be made sense of (see Parkinson \& Manstead, 1993)? In my view, we do not need to have inferred that someone else is to blame in order to get angry, because we can be pushed into an angry 
stance by the diachronically and synchronically structured forces of an ongoing social situation (as in Stemmler's 1989 study, described above). Relatedly, according to Scherer's (e.g., 2001) model of the emotion process, a series of stimulus evaluation checks progressively consolidates a patterned emotional response. At no stage in this process is it necessary to apply a propositional representation of what is happening. Indeed, many of the checks may proceed at the sensorimotor level (Leventhal \& Scherer, 1987).

I agree with Scherer that emotion causation can be a cumulative and piecemeal process. However, in my view, emotions may start out not as sequential responses to information but as ongoing adjustments attuned to unfolding transactions with the practical and social world (Parkinson, 2001). Emotion, like emotion inference, is often specified by the relational stance adopted by actors not by their registration of successive stimuli. The fundamental difference between the two processes, however, is that the former does not necessarily depend on constructing representations of events in either propositional or nonpropositional form. Although emotion in some sense conveys a relational meaning, the person experiencing it does not need to have extracted this meaning before (or even while) becoming emotional.

\section{Resumé}

Siemer and Reisenzein apparently believe that specifically appraisal-relevant information must be accessed before a distinct emotion emerges from an unfolding transaction. For them, there is no alternative route from situation to emotion. But this partly depends on thinking of situations as unfolding separately from our engagement with them (like sentences presented in an experiment), whereas in fact our pattern of engagement can itself specify emotion (in distinction to self-attribution of emotion).

The knowledge-compilation hypothesis implies that humans learn to infer emotions from situations after repeated experience of extracting appraisal information. In other words, 
propositional appraisal representations take ontogenetic priority over discrete emotions. However, these propositional appraisals later drop out of the picture, becoming automatized (or bypassed) with repeated experience. Ultimately, situations come to elicit emotions and situation descriptions come to activate emotion inferences without any explicit mediation by appraisal, and the explicit appraisals, once so familiar that they became automatic, are later only retrievable with special effort.

This seems a roundabout way of explaining the pattern of findings reported in these studies. To me, it gets things back to front. Appraisal judgements are slower and more difficult than emotion judgements because we only learn to make the former after extensive experience with the latter. Propositional appraisals may ultimately come to mediate (some) emotions, but we didn't first come to experience emotions by extracting explicit appraisal information. Like the real-time consolidation of emotion, emotional development is a cumulative process wherein simpler affective responses are combined with relevant action patterns and cognitions. Only when children come to appreciate the rules surrounding their emotional experiences do they start to formalize relational meanings in appraisal-like terms. And only then can they take an indirect route from situations to emotions via appraisals. 


\section{References}

Anderson, J. R. (1987). Skill acquisition: Compilation of weak-method problem solutions. Psychological Review, 94, 192-210.

Barrett, K. C. (1995). A functionalist approach to shame and guilt. In J. P. Tangney \& K. W. Fischer (Eds.), Self-conscious emotions (pp. 25-63). New York: Guilford Press.

Kuppens, P., Van Mechelen, I., Smits, D. J. M., \& De Boeck, P. (2003). The appraisal basis of anger: Specificity, necessity and sufficiency of components. Emotion, 3, 254-269

Lazarus, R. S. (1991). Emotion and adaptation. Oxford: Oxford University Press.

Leventhal, H., \& Scherer, K. (1987).The relationship of emotion to cognition: A functional approach to a semantic controversy. Cognition and Emotion, 1, 3-28.

Parkinson, B. (1999). Relations and dissociations between appraisal and emotion ratings of reasonable and unreasonable anger and guilt. Cognition and Emotion, 13, 347-385.

Parkinson, B. (2001). Putting appraisal in context. In K. R. Scherer, A. Schorr, \& T. Johnstone (Eds.), Appraisal processes in emotion: Theory, methods, research (pp. 173-186). London: Oxford University Press.

Parkinson, B., Fischer, A. H., \& Manstead, A. S. R. (2005). Emotion in social relations: Cultural, group, and interpersonal processes. Philadelphia, PA: Psychology Press.

Parkinson, B., \& Manstead, A. S. R. (1993). Making sense of emotion in stories and social life. Cognition and Emotion, 7, 295-323.

Scherer, K. R. (2001). Appraisal considered as a process of multilevel sequential checking. In K. R. Scherer, A. Schorr \& T. Johnstone (Eds.), Appraisal processes in emotion: Theory, methods, research (pp. 92-120). New York, NY, US: Oxford University Press.

Schuster, B., Rudolph, U., \& Försterling, F. (1998). What determines behavioral decisions? Comparing the role of covariation information and attributions. Personality and Social Psychology Bulletin, 24, 838-854. 
Smith, E. R., \& Miller, F. D. (1983). Mediation among attributional inferences and comprehension processes: Initial findings and a general method. Journal of Personality and Social Psychology, 44, 492-505.

Stemmler, G. (1989). The autonomic differentiation of emotions revisited: Convergent and discriminant validation. Psychophysiology, 26, 617-632.

Vallacher, R. R., Wegner, D. M., \& Somoza, M. P. (1989). That's easy for you to say: Action identification and speech fluency. Journal of Personality and Social Psychology, 56, 199-208.

Watson, J. B. (1929). Psychology from the standpoint of a behaviorist (3rd edition).

Philadelphia: Lippincott. 
Author Note

Work on this commentary was supported by the HUMAINE network of excellence funded by the European Union's Sixth Framework Programme. 


\section{Footnotes}

One trivial reason might be that participants first have to work out which aspect of the described event is referred to in indexical appraisal questions such as "I experience this as undesirable", whereas all the emotion questions had more obvious reference points. A similar problem applies to first-position judgements in studies 2 and 3 . Here cue words were used instead of appraisal and emotion questions, but emotion cue words were generally more self-explanatory (e.g., "anger," "guilt”) than appraisal cue words (e.g., "self," "concerns other/s").

The comparison of these two effects is based on difference scores and carries all the usual associated problems. Although decreases in latencies of appraisal judgements following emotion judgements were greater than decreases in latencies of emotion judgements following appraisal judgements, appraisal judgements had significantly higher latencies in the first place, leaving greater scope for their reduction. Further, no evidence is presented that proportional (rather than absolute) reductions in latency were significantly greater for appraisal judgements. Appraisal judgements facilitated guilt judgements more consistently than judgements of other emotions, and this was the emotion with the longest latencies in the first place. Several situation descriptions used to convey guilt had a similar two-part structure: the protagonist first did something to harm someone, then that other person suffered the consequences (see Siemer and Reisenzein's Appendix A). Participants may have inferred that they needed to have hostile motivations to hurt another person in the first place. This observation may help to explain why participants responded to guilt descriptions with self-attributions of anger in more than $50 \%$ of cases in study 1 . 